\title{
Discontinuous conductance of bichromatically ac-gated quantum wires
}

\author{
Tomasz Kwapiński, ${ }^{1,2, \text { * }}$ Sigmund Kohler, ${ }^{1}$ and Peter Hänggi ${ }^{1}$ \\ ${ }^{1}$ Institut für Physik, Universität Augsburg, Universitätsstraße 1, D-86135 Augsburg, Germany \\ ${ }^{2}$ Institute of Physics, M. Curie-Sktodowska University, 20-031 Lublin, Poland
}

(Dated: December 1, 2018)

\begin{abstract}
We study the electron transport through a quantum wire under the influence of external timedependent gate voltages. The wire is modelled by a tight-binding Hamiltonian for which we obtain the current from the corresponding transmission. The numerical evaluation of the dc current reveals that for bichromatic driving, the conductance depends sensitively on the commensurability of the driving frequencies. The current even possesses a discontinuous frequency dependence. Moreover, we find that the conductance as a function of the wire length oscillates with a period that depends on the ratio between the driving frequencies.
\end{abstract}

PACS numbers: 05.60.Gg, 73.23.-b, 73.63.Nm

\section{INTRODUCTION}

Recent advancements in nanotechnology enabled the investigation of low-dimensional systems like quantum dots, quantum wires or few-atom systems. Thereby, techniques like mechanically controlled break junctions, scanning tunneling microscopy, or angle-resolved photo-emission electron spectroscopy provide information about the electronic structure or transport properties of these systems. Particularly interesting are the transport properties of low-dimensional nanoscale conductors in the presence of time-dependent external fields for which a wealth of effects has been predicted like, e.g., photon-assisted tunneling,, 2.2 and electron pumping, $\underline{3.4}$ External fields can be also used to control both the dc current $t^{\underline{5}}$ and the zero-frequency noise ${ }^{6.7}$ in mesoscopic conductors.

In undriven one-dimensional quantum wires, conductance oscillations - the oscillatory dependence of the conductance as a function of the wire length were predicted $\stackrel{8,9}{\underline{9}}$ and experimentally confirmed $\stackrel{10}{\underline{10}}$ They emerge when the energy levels of a quantum wire match the Fermi energy of the system and relate to charge neutrality of the wire $\underline{11}$ The period of these oscillations is usually two sites for undriven wires, but may become larger if the wire is exposed to a time-dependent field $\underline{12,13,14}$

A second harmonic added to an ac field that drives a quantum wire may significantly alter transport properties. The main reason of the differences is that the second harmonic may brake time-reversal symmetry and generalized parity dynamically, leading to effects like nonadiabatic electron pumping, $\stackrel{15,16,17,18,19}{1}$ i.e., to a dc current even in the absence of any net bias. However, if the ac field acts as a gate voltage that shifts all wire levels uniformly, the pump current must vanish $\frac{16}{} \mathrm{Sim}-$ ilar effects have been predicted for quantum Brownian motion in bichromatically driven infinitely extended periodic potentials ${ }^{20,21,22}$ Moreover, two incommensurable rectangular-shaped driving forces can cause a dc current $^{23,24}$ and lead to interaction-induced frequency mixing, ${ }^{25}$ Also dynamical localization effects in quantum dots may be affected by bichromatic driving fields, in particular when the freuquencies of the latter are commensurable.$^{26,27}$

A well established theoretical tool for treating periodically driven quantum system is Floquet theory, which is based on the discrete time-translation invariance of the driving field. Higher harmonics of the fundamental driving frequency do not affect this invariance and, thus, can be included straightforwardly. As soon as the ratio between the fundamental frequency and its higher harmonic increases, a numerical treatment may require quite some effort. The situation becomes even worse, when the frequencies are no longer commensurable. Then it is rather desirable, even from a practical point of view, to find analytical expressions for the behavior of the driven quantum system, as for example in the recent works 20,21,22. For the description of electron transport through monochromatically driven systems, common transport theories have been combined with Floquet theory $\underline{16,28,29,30}$

In this paper we study the transport properties of a bichromatically driven quantum wire, focussing on the influence of commensurability of the driving frequencies. In doing so, we generalize in Sec. II recent theoretical approaches such that they allow one to treat two ac fields with arbitrary frequency ratio. Then we investigate in Sec. III the influence of bichromatic driving on the transmission and the conductance oscillations.

\section{THEORETICAL DESCRIPTION}

\section{A. Wire-lead model}

We consider the time-dependent wire-lead Hamiltonian $H(t)=H_{\text {wire }}(t)+H_{\text {leads }}+H_{\text {coupl }}$, where

$$
H_{\text {wire }}(t)=\sum_{n=1}^{N}\left[\varepsilon_{n}+f(t)\right] c_{n}^{+} c_{n}+\sum_{n=1}^{N-1} V_{n} c_{n}^{+} c_{n+1}+\text { h.c. }
$$


models in a tight-binding description the wire with sites $|n\rangle$, where neighboring sites are coupled via tunnel matrix elements $V_{n}$. The on-site energies $\varepsilon_{n}$ are modulated by an ac gate voltage $f(t)$. The first and the last site of the wire are coupled via the tunnel Hamiltonian

$$
H_{\text {coupl }}=\sum_{\vec{k} L} V_{\vec{k} L} c_{\vec{k} L}^{+} c_{1}+\sum_{\vec{k} R} V_{\vec{k} R} c_{\vec{k} R}^{+} c_{N}
$$

to leads at the left $(L)$ and at the right $(R)$ end. Within a wide-band approximation, we assume that the spectral density of the wire-lead coupling, $\Gamma_{\alpha}(\varepsilon) \equiv$ $2 \pi \sum_{\vec{k}}\left|V_{\vec{k}}\right|^{2} \delta\left(\varepsilon-\varepsilon_{\alpha}\right)=\Gamma_{\alpha}$, is energy independent. The leads are modelled as ideal Fermi gases by the Hamiltonian

$$
H_{\text {leads }}=\sum_{\alpha=L, R} \sum_{\vec{k}} \varepsilon_{\vec{k} \alpha} c_{\vec{k} \alpha}^{+} c_{\vec{k} \alpha}
$$

The operators $c_{\vec{k} \alpha}, c_{n}$, and their Hermitian adjoints are the usual annihilation and creation operators for an electron in lead $\alpha=L, R$ with wave vector $\vec{k}$ and for an electron at the $n$th wire site, respectively. We focus on experiments like those described in Refs. 9, 31,32, in which electron-electron interactions can be neglected. For photon-assisted transport, this is, e.g., the case when the photon energy is much smaller than both the typical splitting between single-particle energies of the wire and the Coulomb interaction. 33

\section{B. Time-dependent scattering theory}

Following the scattering approach of Ref. 16, we derive from the time-dependent wire-lead Hamiltonian $H(t)$ the Heisenberg equations of motion for the creation operators. The wire part of their solution can be expressed in terms of a retarded Green function $G\left(t, t^{\prime}\right)$ which, owing to the time-dependence of the Hamiltonian, depends explicitly on both times and fulfills the equation of motion

$$
\left[i \hbar \frac{d}{d t}-H_{0}-f(t)+\frac{i}{2} \Gamma\right] G\left(t, t^{\prime}\right)=\delta\left(t-t^{\prime}\right) .
$$

The Hamiltonian $H_{0}=H_{\text {wire }}-f(t) \mathbf{1}_{\text {wire }}$ describes the wire in the absence of the time-dependent gating, while $\Gamma=\Gamma_{L}|1\rangle\left\langle 1\left|+\Gamma_{R}\right| N\right\rangle\langle N|$ is a self-energy stemming from the coupling of the wire to the leads.

The solution of Eq. (4) can be traced back to the solution of the time-independent problem for which the Green function $g$ obeys the equation of motion

$$
\left[i \hbar \frac{d}{d t}-H_{0}+\frac{i}{2} \Gamma\right] g\left(t-t^{\prime}\right)=\delta\left(t-t^{\prime}\right)
$$

Then it is straightforward to show that the Green functions $G$ and $g$ are related according to

$$
G\left(t, t^{\prime}\right)=e^{-i\left[F(t)-F\left(t^{\prime}\right)\right]} g\left(t-t^{\prime}\right) .
$$

This means that the influence of the driving field is subsumed in the phase

$$
F(t)=\int^{t} d s f(s)
$$

Defining the current through the wire as the change of the electron number in the left lead, $I=-e d n_{L}(t) / d t$, we obtain for its expectation value the time-dependent Landauer-like expression

$$
I(t)=\frac{e}{h} \int d \varepsilon\left[T_{R L}(t, \varepsilon) f_{L}(\varepsilon)-T_{R L}(t, \varepsilon) f_{L}(\varepsilon)\right]-\dot{q}_{L}(t)
$$

with the time-dependent transmission

$$
T_{L R}(t, \varepsilon)=\Gamma_{L} \Gamma_{R}\left|G_{1, N}(t, \varepsilon)\right|^{2}
$$

and $T_{L R}$ defined accordingly. The function

$$
\begin{aligned}
G(t, \varepsilon) & =\int d \tau e^{i \varepsilon \tau} G(t, t-\tau) \\
& =e^{-i F(t)} \int d \tau e^{i \varepsilon \tau+i F(t-\tau)} g(\tau)
\end{aligned}
$$

is the Fourier transformed of the Green function with respect to the time difference $\tau=t-t^{\prime}$. Note that $G$ still depends explicitly on the final time $t$. The displacement current $\dot{q}_{L}(t)$ describes charges oscillating between the left lead and the wire and does not contribute to the dc current considered below. Thus it will not be considered henceforth.

In order to evaluate the $\tau$-integration in Eq. (11), we have to specify the time-dependent gate voltage $f(t)$. We assume that it consists of $K$ harmonics, such that

$$
\begin{aligned}
& f(t)=\sum_{k=1}^{K} \Delta_{k} \cos \left(\omega_{k} t\right), \\
& F(t)=\sum_{k=1}^{K} \frac{\Delta_{k}}{\omega_{k}} \sin \left(\omega_{k} t\right),
\end{aligned}
$$

where $\omega_{k}$ and $\Delta_{k}$ are the frequency and the amplitude of the $k$ th harmonic of the ac field. Then the Green function (11) reads

$$
\begin{aligned}
G(t, \varepsilon)= & \sum_{m_{1}, \cdots, m_{K}} J_{m_{1}}\left(\Delta_{1} / \omega_{1}\right) \cdots J_{m_{K}}\left(\Delta_{K} / \omega_{K}\right) \\
& \times \exp \left(i \sum_{k} m_{k} \omega_{k} t\right) g\left(\varepsilon-\sum_{k} m_{k} \omega_{k}\right) .
\end{aligned}
$$

The Bessel functions $J_{m}$ stem from a Fourier decomposition of the exponential in Eq. (6), while the evaluation of the $\tau$ integral provided the Fourier transformed of the Green function, $g(\epsilon)$. Note that we have ignored a timedependent but nevertheless irrelevant phase factor.

Assuming moreover symmetric wire-lead coupling, i.e., $\Gamma_{L}=\Gamma_{R}=\Gamma$, the time-dependent transmission (9) be- 
comes

$$
\begin{aligned}
T_{L R}(t, \varepsilon)= & \Gamma^{2} \sum_{m_{1}, \cdots, m_{K}} J_{m_{1}}\left(\Delta_{1} / \omega_{1}\right) \cdots J_{m_{K}}\left(\Delta_{K} / \omega_{K}\right) \\
& \times \sum_{m_{1}^{\prime}, \cdots, m_{K}^{\prime}} J_{m_{1}^{\prime}}\left(\Delta_{1} / \omega_{1}\right) \cdots J_{m_{K}^{\prime}}\left(\Delta_{K} / \omega_{K}\right) \\
& \times g_{1 N}\left(\varepsilon-\sum_{k} m_{k} \omega_{k}\right) g_{1 N}^{*}\left(\varepsilon-\sum_{k} m_{k}^{\prime} \omega_{k}\right) \\
& \times \exp \left(i \sum_{k}\left(m_{k}-m_{k}^{\prime}\right) \omega_{k} t\right) \\
= & T_{R L}(t, \varepsilon) .
\end{aligned}
$$

The relation in the last line means that from the right lead to the left lead has the same probability as the reversed process. This is particular for ac gating and does not hold for general driving. ${ }^{16}$ Here it stems from the fact that for the present model with time-dependent gating, the time-dependent transmission can be expressed in terms of the Green function $g$ for the time-independent problem.

The dc current, being the main quantity of interest, is then obtained from Eq. (8) and reads

$$
I_{0}=\frac{e}{h} \int d \varepsilon\left[f_{L}(\varepsilon)-f_{L}(\varepsilon)\right] T(\varepsilon)
$$

where $T(\varepsilon)$ denotes the time-average of the transmission $T_{L R}(t, \varepsilon)$, and its computation from Eq. (15) seems straightforward. This is however not the case, because the averaging procedure depends crucially on the commensurability of the frequencies $\omega_{k}$. If the gate voltage $f(t)$ consists of many spectral components, the actual computation can be quite cumbersome. However, we below restrict ourselves to two frequencies, so that it is sufficient to address two cases: all frequencies being commensurable or all being incommensurable.

For incommensurable frequencies, the phase factor in the last line of the time-dependent transmission (15) vanishes if and only if $m_{k}=m_{k}^{\prime}$ for each mode $k$. Consequently, we obtain the average transmission

$$
\begin{aligned}
T(\varepsilon)= & \sum_{m_{1}, \cdots, m_{K}} \Gamma^{2} J_{m_{1}}^{2}\left(\Delta_{1} / \omega_{1}\right) \cdots J_{m_{K}}^{2}\left(\Delta_{K} / \omega_{K}\right) \\
& \times\left|g_{1 N}\left(\varepsilon-\sum_{k} m_{k} \omega_{k}\right)\right|^{2} .
\end{aligned}
$$

The Green function of the undriven wire, $g(\epsilon)$, depends on the specific wire model and will be determined below.

If the frequencies of the external fields are commensurable, time-averaging unfortunately does not result in a less involved expression for the transmission. Therefore we provide the explicit result only for the case of two frequencies with ratio $\omega_{1} / \omega_{2}=m / m^{\prime}$, where $m$ and $m^{\prime}$ are integers. Then we obtain

$$
\begin{aligned}
T(\varepsilon)=\sum_{<m_{1}, m_{2}, m_{1}^{\prime}, m_{2}^{\prime}>} & \Gamma^{2} J_{m_{1}}\left(\Delta_{1} / \omega_{1}\right) J_{m_{2}}\left(\Delta_{2} / \omega_{2}\right) \\
& \times J_{m_{1}^{\prime}}\left(\Delta_{1} / \omega_{1}\right) J_{m_{2}^{\prime}}\left(\Delta_{2} / \omega_{2}\right) \\
& \times g_{1 N}\left(\varepsilon-m_{1} \omega_{1}-m_{2} \omega_{2}\right) \\
& \times g_{1 N}^{*}\left(\varepsilon-m_{1}^{\prime} \omega_{1}-m_{2}^{\prime} \omega_{2}\right)
\end{aligned}
$$

where the angular brackets restrict the sum to those values of $m_{1}, m_{2}, m_{3}, m_{4}$ that comply with the condition $\left(m_{1}-m_{1}^{\prime}\right) \omega_{1}=-\left(m_{2}-m_{2}^{\prime}\right) \omega_{2}$. Note that for commensurable frequencies, this condition can also be fulfilled for $m_{k} \neq m_{k}^{\prime}$. Since for incommensurable frequencies the restriction to the sum is fulfilled only for $m_{1}=m_{1}^{\prime}$ and $m_{2}=m_{2}^{\prime}$, expression (19) incidentally holds for both cases.

The transmissions (18) and (19) represent the main analytical results of this work. In the case of commensurable frequencies, the driving is periodic and, thus, can be treated within Floquet scattering theory. 6.12,16 Nevertheless, the explicit evaluation of the transmission can be quite cumbersome. The case of incommensurable frequencies generally even requires a "two-color Floquet theory" which can be numerically demanding. Only the restriction to models with uniform gating allowed us to obtain the present compact expressions for the transmission.

\section{WIRE WITH EQUAL ON-SITE ENERGIES}

In order to present explicit results, we have to specify the wire model. A $N$-site model that allows even further analytical treatment is one with equal onsite energies $\varepsilon_{n}=\varepsilon_{0}$ for all sites $|n\rangle, n=1, \ldots, N$ and equal couplings $V_{n}=V$. For such a wire, the relevant element of the Green function in the absence of driving reads

$$
g_{1 N}(\varepsilon)=\frac{(-1)^{N-1}}{V u_{N}(x)+i \Gamma u_{N-1}(x)-\Gamma^{2} u_{N-2}(x) / 4 V},
$$

where $x=\left(\varepsilon-\varepsilon_{0}\right) / 2 V$, while $u_{n}$ denotes the $n$th Chebyschev polynomial of the second kind. For a derivation, we refer the reader to Ref. 34. This relation together with Eqs. (18) and (19) allows one to obtain explicit expressions for the transmission of the ac-gated wire (11).

In our calculations we use the effective wire-lead coupling strength $\Gamma$ as energy unit. Then for $\Gamma=0.1 \mathrm{eV}$, the current unit becomes $2 e \Gamma / \hbar \simeq 50 \mu \mathrm{A}$. Moreover, we measure level energies with respect to the Fermi energy, which implies $E_{F}=0$. Henceforth, we present results for a bichromatic driving field. 


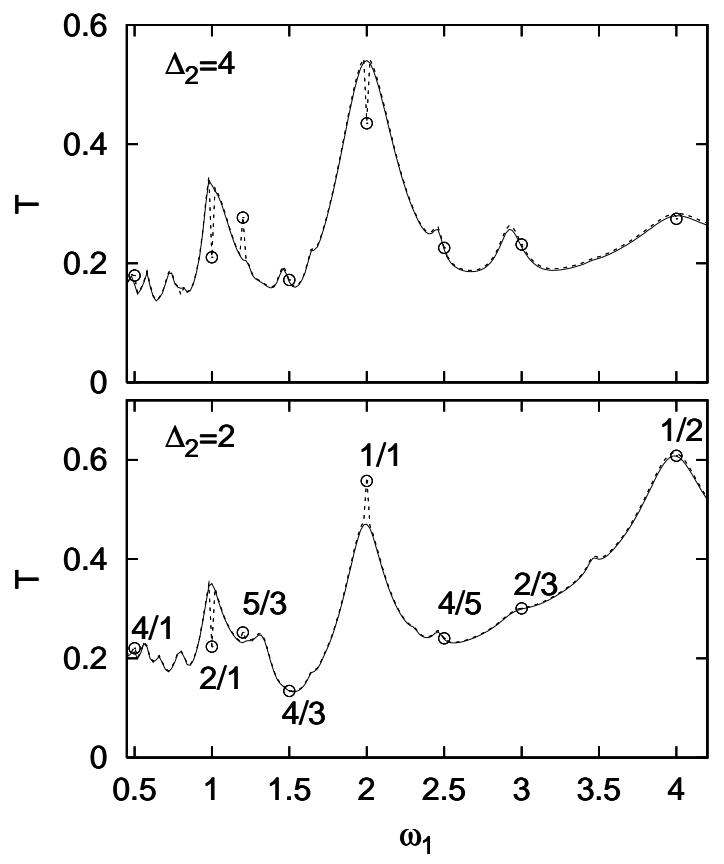

FIG. 1: Transmission for a wire with $N=5$ sites with equal onsite energies $\varepsilon_{0}=0$ as a function of the frequency $\omega_{1}$ for the driving amplitudes $\Delta_{2}=4$ (a) and $\Delta_{2}=2(\mathrm{~b})$. The tunnel coupling between two neighboring wire sites is $V=4$, while the other driving parameters read $\omega_{2}=2, \Delta_{1}=4$. Solid lines are obtained from expression 18 which is valid for incommensurable frequencies only, while the dashed lines and the circles are obtained from Eq. (19) and, thus, are valid for all driving frequencies $\omega_{1}, \omega_{2}$. The fractions in panel (b) mark selected frequency ratios $\omega_{2} / \omega_{1}$.

\section{A. Discontinuous transmission and corresponding current}

In order to reveal the role of commensurability of the driving frequencies, we investigate the transmission of a wire with onsite energies at the Fermi energy level. Figure 1 shows the result as a function of the frequency of the first external field, $\omega_{1}$, while the frequency of the second field is kept at a constant value. As a most prominent feature, the transmission turns out to be a discontinuous function, which assumes exceptional values whenever $\omega_{2} / \omega_{1}$ is rational, i.e., when the driving frequencies are commensurable. A clearly visible effect is found for ratios that can be expressed by small natural numbers. In particular, for the ratios $1 / 1$ and $2 / 1$, the deviation from the transmission in the vicinity of the resonance is of the order of $20 \%$. For "less rational" numbers like e.g. $4 / 3$ or $2 / 3$, we still find a small discontinuity which, however, is hardly visible on the scale chosen. The values can depend sensitively on details like driving amplitudes and wire parameters. In the present case, it is even such that the deviation at the prime resonance $1 / 1$ turns with increasing amplitude $\Delta_{2}$ from a remarkable negative value to a positive value; cf. panels (a) and (b) of Fig. 1. More-

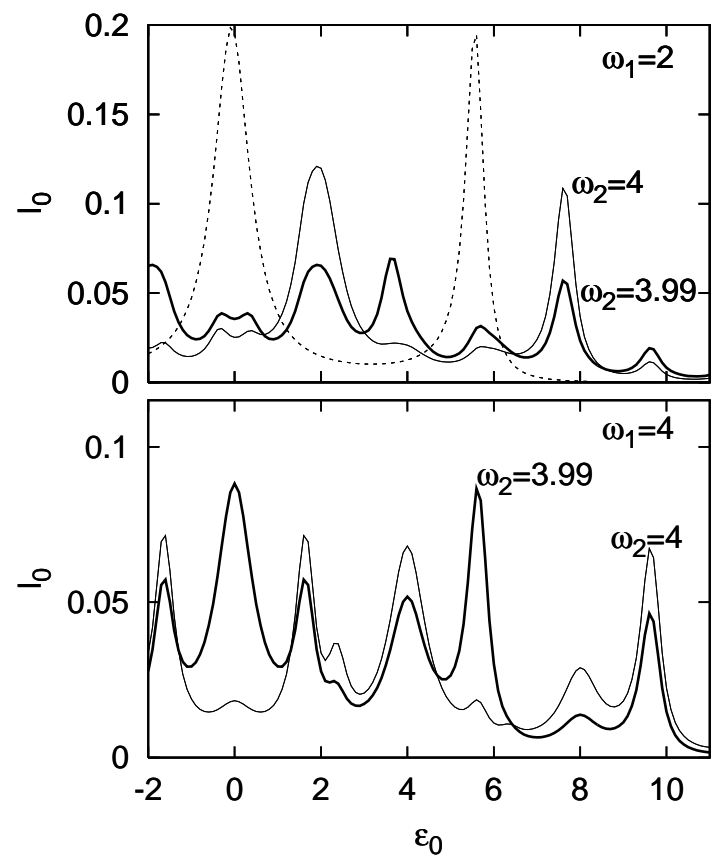

FIG. 2: Average current for the chemical potentials $\mu_{L}=$ $-\mu_{R}=0.1$ as a function of the equal onsite energies $\varepsilon_{0}$ for a wire with $N=3$ sites and tunneling matrix elements $V=4$. The first driving field is kept constant $\left[\Delta_{1}=4\right.$ with frequency $\omega_{1}=2(\mathrm{a})$ and $\left.\omega_{1}=4(\mathrm{~b})\right]$. The second field has driving amplitude $\Delta_{2}=4$, while its frequency is $\omega_{2}=4$ (thick solid lines) and $\omega_{2}=3.99$ (thin solid lines), respectively. The dashed line in panel (a) marks the undriven case which corresponds to vanishing driving amplitudes, i.e. $\Delta_{1}=\Delta_{2}=0$.

over, the $2 / 1$ resonance, albeit "very rational", does here not lead to a significant discontinuity. A similar behavior has been observed for the transport in classical ratchet devices $\stackrel{24}{=}$ It is worth noting that the transmission for incommensurable frequencies [Eq. (18) and solid lines in Fig. 1. possesses local maxima at the resonances $1 / 2,1 / 1$, and $2 / 1$.

In order to investigate further the role of commensurability, we focus on the $2 / 1$ resonance for $\omega_{1}=2$ and the $1 / 1$ resonance for $\omega_{1}=4$. In doing so, we compare the current for the commensurable case $\omega_{2}=4$ with the one found for the "less commensurable" $\omega_{2}=3.99$. The current for a wire of length $N=3$ as a function of the onsite energy $\varepsilon_{0}$ is shown in Fig. 2. A common feature of both cases is the emergence of side peaks: The undriven wire possesses the eigenenergies $E_{0}=\varepsilon_{0}$ and $E_{ \pm}=\varepsilon_{0} \pm 5.6$. Whenever these eigenenergies lie within the voltage window at the Fermi energy $E_{F}=0$, i.e. for $\varepsilon_{0}=0$ and for $\varepsilon_{0}=\mp 5.6$, the current assumes a maximum in compliance with the Breit-Wigner formula; see Fig. 1 of Ref. 34 .

In the presence of ac gating, we find side peaks shifted from the original peaks by multiples of the driving frequencies $\omega_{1}$ and $\omega_{2}$. Overlapping peaks may lead to destructive interference. This is, e.g., the case for the data shown in Fig. 2(a) in the vicinity of $\varepsilon_{0}=0$, where the 
side peaks at $\varepsilon_{0} \mp \omega_{1} \mp \omega_{2}$ for $\varepsilon_{0}= \pm 5.6$ compensate the fundamental peak at $\varepsilon_{0}=0$, such that the total current is characterized by the local minimum. The proper location of the side peaks is most clearly visible for $\omega_{1}=4$ and $\omega_{2} \approx 4$ [Fig. 2(a)], i.e., when both frequencies are practically indistinguishable. Then we observe side peaks at $\varepsilon_{0}=4,8$ (main peak at $\varepsilon_{0}=0$ ), at $\varepsilon_{0}=1.6,9.6$ (main peak at $\varepsilon_{0}=5.6$ ), and at $\varepsilon_{0}=2.4$ (main peak at $\left.\varepsilon_{0}=-5.6\right)$. Let us emphasize that the location of the peaks does not allow us to divide the commensurable case from the incommensurable case, because the resonance width $\Gamma=1$ is much larger than the difference between the two values for $\omega_{2}$ which reads $4-3.99=0.01$.

Despite the practically identical location of the peaks, the peak heights are significantly different for the two cases. This corresponds to the discontinuity observed already in Fig. 1. The difference can even be up to a factor 5 , as is the case for $\omega_{1}=4$ [Fig. 2(b)] for the zeroth-order resonances at $\varepsilon_{0}=0$ and $\varepsilon_{0}=5.6$. This means that in a possible experiment, the current may be controlled via a slight shift of one of the driving frequencies, provided that one tunes the wire parameters such that the current in the absence of the driving assumes a large value.

\section{B. Conductance oscillations}

The conductance $G$ of a tight-binding system with onsite energies at the Fermi level obeys oscillations as a function of the wire length. Typically these oscillations have period 2 (even-odd oscillations), $, 9,9,10,11$ but also larger periods have been predicted for both static $34,35,36,37$ and monochromatically driven $\frac{12}{}$ wires. We here investigate the corresponding behavior for bichromatic gating.

The oscillation period for the driven case can be estimated along the lines followed in Ref. 12: For a static wire, the emergence of conductance oscillation with period $M$ means that a wires with $N$ sites possesses the same conductance as a wire with $N+M$ sites, i.e.

$$
g_{1, N}(\varepsilon)=g_{1, N+M}(\varepsilon) .
$$

After some algebra, one obtains ${ }^{34} \cos (m \pi / M)=(\varepsilon-$ $\left.\varepsilon_{0}\right) / 2 V$, where $m=0,1, \ldots, M-1$. The energy difference on the right-hand side of this equation marks the distance of the onsite energies $\varepsilon_{0}$ to the Fermi energy of the leads attached. In the presence of ac fields, onsite energies acquire shifts by multiples of the driving field quanta. Then the oscillation period is determined by the condition

$$
\cos \left(\frac{m \pi}{M}\right)=\frac{\varepsilon-\varepsilon_{0}+k_{1} \omega_{1}+k_{2} \omega_{2}}{2 V},
$$

where again $m=0,1, \ldots, M-1$, while $k_{1}$ and $k_{2}$ are integer numbers.

In order to corroborate this condition, we have computed the transmission [which relates to the conductance

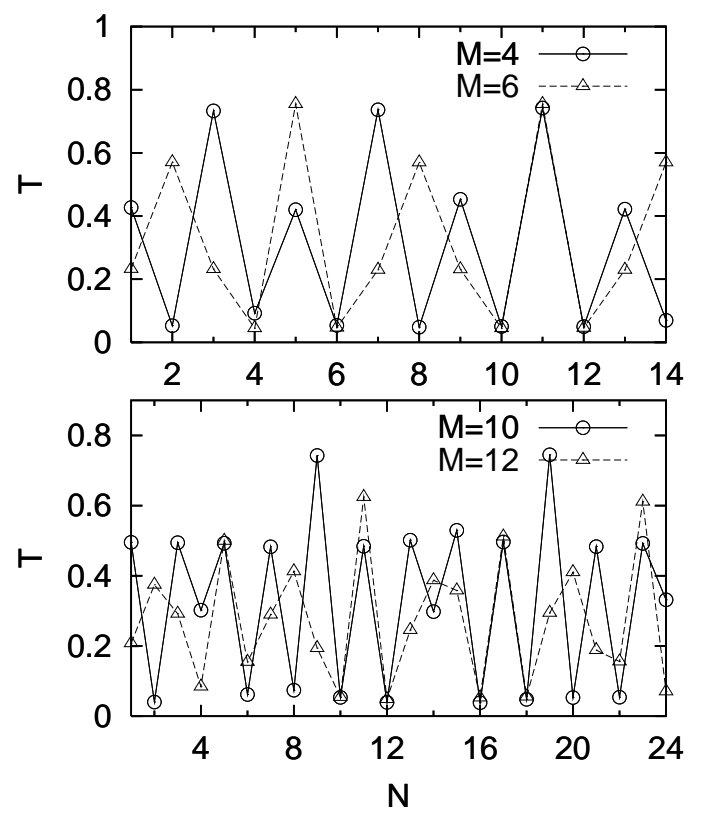

FIG. 3: Transmission as a function of the wire length $N$ for $\Delta_{1}=\Delta_{2}=6, \varepsilon_{0}=0, V=4$. (a) the driving frequencies are $\omega_{1}=\sqrt{2} \mathrm{~V}, \omega_{2}=2 \mathrm{~V}$ (circles) and $\omega_{1}=V, \omega_{2}=2 \mathrm{~V}$ (triangles) correspond to the oscillation periods $M=4$ and $M=6$, respectively. (b) $\omega_{1}=2 V \cos (\pi / 5), \omega_{2}=2 V$ (circles) and $\omega_{1}=V, \omega_{2}=\sqrt{2} V$ (triangles) corresponding to $M=10$ and $M=12$, respectively. The lines serve as a guide to the eye.

according to $\left.G=\left(2 e^{2} / h\right) T\right]$ for bichromatic driving. The results for various combinations of the driving frequencies are shown in Fig. 3. For all parameters used in the numerical evaluation of the transmission (19), we observe an oscillation period $M>2$ in compliance with Eq. (22). Conductance oscillations with period $M=6$ have also been found in Ref. 12 for wires under the influence of a monochromatic ac field. There the oscillation period is also determined by Eq. (22) for $k_{2}=0$. We like to emphasize that in the absence of the driving, by contrast, the oscillation period is $M=2$, i.e., we find the usual even-odd oscillations.

A remaining intriguing question is whether for bichromatic driving, the commensurability of the frequencies influences the conductance oscillations. Therefore, we compare for $\omega_{1}=V$ the commensurable $\omega_{2}=\omega_{1}$ (which in fact represents monochromatic driving) with the "practically incommensurable" $\omega_{2}=0.99 \omega_{1}$. For both cases, Eq. (22) predicts conductance oscillations with a period of approximately $M=6$. Figure 4 depicts the resulting transmission as a function of the wire length. Note, that the oscillation period for the static conductor is $M=2$, while it becomes $M=6$ in the presence of the driving. Generally, the variation of the conductance within one oscillation period is larger for incommensurate driving frequencies. For the driving parameters used in Fig. 4 it is even such that for the com- 

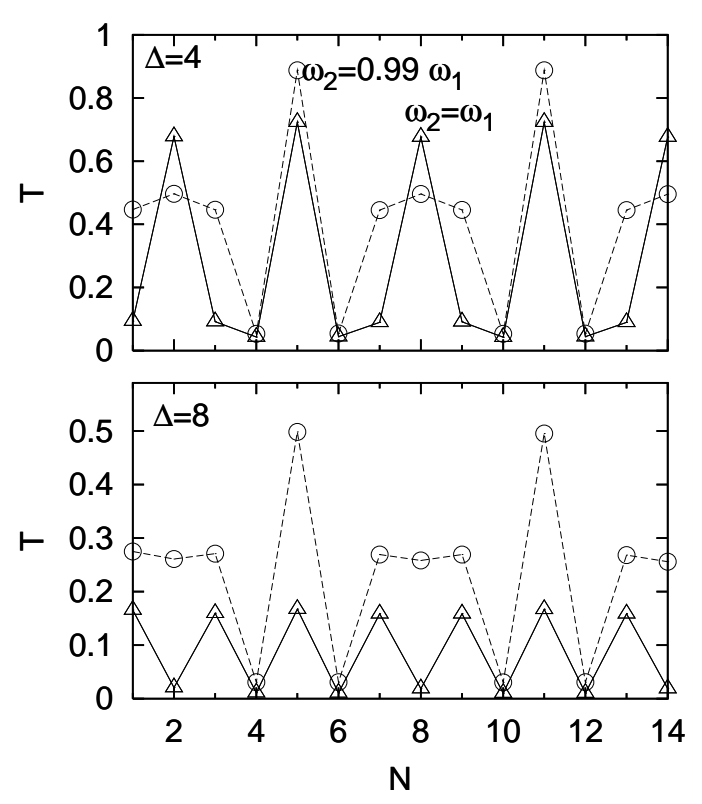

FIG. 4: Transmission as a function of the wire length $N$ for commensurable frequencies $\left(\omega_{1}=\omega_{2}=4\right.$, triangles $)$ and incommensurable frequencies $\left(\omega_{2}=0.99 \omega_{1}, \omega_{1}=4\right.$, circles $)$ and the driving amplitudes $\Delta_{1}=\Delta_{2}=\Delta=4$ (a) and $\Delta_{1}=\Delta_{2}=\Delta=8(\mathrm{~b})$. The other parameters are $\varepsilon_{0}=0$, $V=4$. The lines are a guide to the eye.

mensurate $\omega_{1}=\omega_{2}$, the oscillations have almost period $M=3$ or $M=2$ (solid lines) with a small variation of every second peak, such that strictly speaking, the period is still $M=6$. Moreover, we again observe one significant difference between the commensurate and the incommensurate case: For given driving amplitudes, the conductance may change by almost one order of magnitude upon shifting the second frequency from $\omega_{2}=\omega_{1}$ to $\omega_{2}=0.99 \omega_{1}$. This again allows one in an experiment to switch the current by changing one driving frequency by only $1 \%$.

Note also that when the driving amplitude becomes smaller, the situation tends towards the undriven limit for which the oscillation period is $M=2$. The transition is such that the conductance within the $M=6$ period changes until eventually the oscillation period becomes practically $M=2$.

\section{CONCLUSIONS}

Using a Green's function technique, we have derived a Landauer-like formula for the current through a poly- chromatically ac-gated quantum wire described by a tight-binding model. If the gating acts uniformly on all wire sites, we have provided an expression for the timedependent transmission that depends on the transmission in the absence of the driving and some time-dependent prefactors. The time-average of the transmission for ac gating determines the dc current being the central experimentally accessable quantity. In order to perform the time-average, we had to distinguish between commensurable and incommensurate frequencies. We have found that the transmission and, thus, the dc current depends sensitively on the commensurability of the driving frequencies. The difference is most noticeable close to the resonances with the "very rational" frequency ratios $1 / 2$ and $1 / 1$, where the conductance as a function of one driving frequency is discontinuous and may suddenly change by up to one order of magnitude.

The commensurability of the driving frequencies leaves also its fingerprints in the conductance oscillations: While for static conductors, one usually observes an oscillation period of two sites (even-odd oscillations), bichromatic driving may increase the period significantly. For our model, we observed oscillation periods of up to 12 sites. The physical reason for the enlarged period is that the bichromatic driving influences the resonance condition that determines the oscillations. We have confirmed this intuitive prediction by numerical studies. As a remarkable influence of the commensurability, we found that the growth of the oscillation period is more visible for incommensurate frequencies.

In a possible experiment with STM tips that contact a

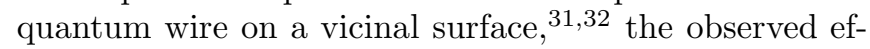
fect may be used for switching the current upon slightly changing the frequency of the ac gate voltage. Alternatively, such an experiment may also be performed with a molecular wire that bridges a break junction 10,38 or with a chain of coherently coupled quantum dots $39,40 \mathrm{In}$ particular the latter type of experiment is well suited for applying time-dependent gate voltages.

\section{Acknowledgments}

This work has been supported by the Grant No. N N202 146833 of the Polish Ministry of Science and Higher Education and the Alexander von Humboldt Foundation (TK). SK and PH acknowledge support by the DFG via SPP 1243, the excellence cluster "Nanosystems Initiative Munich" (NIM), and the German-Israeli Foundation (GIF).
* Electronic address: tomasz.kwapinski@umcs.lublin.pl

1 T. H. Oosterkamp, L. P. Kouwenhoven, A. E. A. Koolen,
N. C. van der Vaart, and C. J. P. M. Harmans, Phys. Rev. Lett. 78, 1536 (1997). 
${ }^{2}$ W. G. van der Wiel, S. De Franceschi and J. M. Elzerman, T. Fujisawa, S. Tarucha, and L. P. Kouwenhoven, Rev. Mod. Phys. 75, 1 (2003).

3 C. A. Stafford and N. S. Wingreen, Phys. Rev. Lett. 76, 1916 (1996).

${ }^{4}$ L. P. Kouwenhoven, A. P. Johnson, N. C. van der Vaart, A. van der Enden, C. J. P. M. Harmans and C. T. Foxon, Z. Phys. B: Condens. Matter 85, 381, (1991).

5 J. Lehmann, S. Camalet, S. Kohler, and P. Hänggi, Chem. Phys. Lett. 368, 282 (2003).

6 S. Camalet, J. Lehmann, S. Kohler, and P. Hänggi, Phys. Rev. Lett. 90, 210602 (2003).

7 S. Camalet, S. Kohler, and P. Hänggi, Phys. Rev. B 70, 155326 (2004).

8 N. D. Lang, Phys. Rev. Lett. 79, 1357 (1997).

9 Z. Y. Zeng and F. Claro, Phys. Rev. B 65, 193405 (2002).

10 R. H. M. Smit, C. Untiedt, G. Rubio-Bollinger, R. C. Segers, and J. M. van Ruitenbeek, Phys. Rev. Lett. 91, 076805 (2003).

11 H.-S. Sim, H.-W. Lee, and K. J. Chang, Phys. Rev. Lett. 87, 096803 (2001).

12 T. Kwapiński, Phys. Rev. B. 69, 153303 (2004).

13 D. F. Martinez and R. A. Molina, Eur. Phys. J 52, 281 (2006).

14 D. F. Martinez, R. A. Molina, and B. Hu, Phys. Rev. B 78, 045428 (2008).

15 J. Lehmann, S. Kohler, P. Hänggi, and A. Nitzan, J. Chem. Phys. 118, 3283 (2003).

16 S. Kohler, J. Lehmann, and P. Hänggi, Phys. Rep. 406, 379 (2005).

17 M. Moskalets and M. Büttiker, Phys. Rev. B 70, 245305 (2004).

18 M. M. Mahmoodian and M. V. Entin, EPL 77, 67002 (2007).

19 L. Arrachea, A. Levy Yeyati, and A. Martin-Rodero, Phys. Rev. B 77, 165326 (2008).

20 I. Goychuk and P. Hänggi, Europhys. Lett. 43, 503 (1998).

21 S. Sengupta, R. Guantes, S. Miret Artes, and P. Hänggi,
Physica A 338, 406 (2004).

22 M. Borromeo, P. Hänggi, and F. Marchesoni, J. Phys.: Condens. Matter 17, S3707 (2005).

23 S. Savelev, F. Marchesoni, P. Hänggi, and F. Nori, Eur. J. Phys. B 40, 403 (2004).

24 S. Savelev, F. Marchesoni, P. Hänggi, and F. Nori, Europhys. Lett. 67, 179 (2004).

25 M. Thorwart, R. Egger, and A. O. Gogolin, Phys. Rev. Lett. 101, 036806 (2008).

26 D. M. Basko, M. A. Skvortsov, and V. E. Kravtsov, Phys. Rev. Lett. 90, 096801 (2003).

27 X.-B. Wang and V. E. Kravtsov, Phys. Rev. B 64, 033313 (2001).

28 C. Bruder and H. Schoeller, Phys. Rev. Lett. 72, 1076 (1994).

29 G. Platero and R. Aguado, Phys. Rep. 395, 1 (2004).

30 L. Arrachea and M. Moskalets, Phys. Rev. B 74, 245322 (2006).

31 M. Krawiec, T. Kwapiński and M. Jałochowski, phys. stat. sol. b 242, 332 (2005).

32 M. Krawiec, T. Kwapiński and M. Jałochowski, Phys. Rev. B 73,075415 (2006).

33 H.-K. Zhao and J. Wang, Eur. Phys. J. B 59, 329 (2007).

34 T. Kwapiński, J. Phys.: Condens. Matt. 17, 5849 (2005).

35 P. L. Pernas, F. Flores, and E. V. Anda, J. Phys.: Condens. Matter 45309 (1992)

${ }^{36}$ K. S. Thygesen, K. W. Jacobsen, Phys. Rev. Lett. 91, 146801 (2003).

37 R. A. Molina, D. Weinmann and J.-L. Pichard, Europhys. Lett. 6796 (2004).

38 N. Agrait, A. Levy Yeyati, J. M. van Ruitenbeek, Phys. Rep. 377, 81 (2003).

39 R. H. Blick, R. J. Haug, J. Weis, D. Pfannkuche, K. von Klitzing, and K. Eberl, Phys. Rev. B 53, 7899 (1996).

40 D. Schröer, A. D. Greentree, L. Gaudreau L, K. Eberl, L. C. L. Hollenberg, J. P. Kotthaus, and S. Ludwig, Phys. Rev. B 76, 075306 (2007). 